\title{
Processi Dinamici nella Cultura Postmoderna: Interpenetrazione tra Pubblicità e Cultura
}

\author{
Ananyeva Evgenia Viktorovna \\ St. Petersburg University of Humanities and Social Sciences, \\ graduate student of Department of Cultural Theory and History, \\ Russia, St. Petersburg \\ eva-1986@inbox.ru
}

\section{Doi:10.5901/mjss.2013.v4n9p318}

\begin{abstract}
Dynamic processes in the culture of Post-Modernism: Interpenetration of advertizing and culture. The work describes dynamic processes taking place in culture of the era of postmodernism which are characterized by a sharp growth of cultural and social diversity, declarations of the plurality principle, fragmentation of the cultural unity, attention to personality and its inner world, self-controlled structures, identity problems, increase in the opportunities for diversity of progress, refusal from some principles of the global social action. Purpose of the research is to establish the causes and mechanisms of the dynamic processes' influence on the modern society through analyzing the era of postmodern culture. Advertizing, as a "mass culture" element, creates an uncritical, consumer consciousness. Advertizing and culture of the postmodern era are in a close interrelation, and have an effect of interpenetration.
\end{abstract}

Keywords: culture of a postmodern, cultural dynamics, deformation of values, mass consumer society, advertising, symbol in advertising

La scelta di questo tema è condizionata dalle tendenze di spostare l'umanità nel nuovo periodo ossia nell'epoca postmoderna. La società moderna si trova in una situazione in cui ha bisogno di trovare certi regolatori dei processi culturali che potrebbero motivare e apprezzare la caratteristica dei cambiamenti delle trasformazioni che stanno avvenendo nella cultura, siccome la società della cultura classica è arrivata a un nuovo livello di sviluppo grazie al potenziamento tecnico-scientifico e alla rivoluzione informatica.

L'obiettivo della ricerca è manifestare le cause ed i meccanismi dell'influenza dei processi dinamici sulla società moderna, analizzando l'epoca della cultura postmoderna.

La rilevanza della ricerca di questo tema proviene prima di tutto dai processi dinamici nella cultura dell'epoca postmoderna caratterizzata da un drastico aumento della varietà culturale e sociale; dalla proclamazione del principio della molteplicità; dalla frammentazione della concordanza culturale; dall'attenzione alla personalità e al suo mondo interiore, alle strutture autogovernate, ai problemi dell'identità con l'aumento delle possibilità della multivarianza del progresso, con il rifiuto di alcuni principi dell'effetto sociale universale.

I cambiamenti impetuosi nella sfera tecnica e tecnologica, il progresso tecnico-scientifico rinnovano radicalmente lo spazio materiale, in cui si svolge direttamente l'attività umana. Lo sviluppo delle scienze della società e della cultura della seconda metà del XX - l'inizio del XXI secolo hanno notato abbastanza distintamente le funzioni programmatorie della cultura e dell' attività vitale umana. Gradualmente è venuto fuori che non esiste un settore di attività, di comportamento e di communicazione tra le persone, non esistono competizioni, strutture, istituti sociali, che apparirebbero, riprodurerebbero e muterebbero fuori l'influenza culturale» (Stepin, V.S. (2011) Civiltà e cultura. San Pietroburgo: SPbGUP. p. 78)

Nei discorsi sulla manifestazione del postmodernismo, si possono riportare le parole di un analista autorevole Umberto Eco: «ll postmodernismo è la risposta al modernismo: visto che il passato non può essere distrutto, perché la sua distruzione porta al silenzio, deve essere rivisitato: con ironia, in modo non innocente ...» (Kagan, M.S. (1997) Estetica come scienza filosofica. San Pietroburgo: TOO TK "Petropolis". p. 523). Nella frase del semiotico italiano sono ben noti degli elementi della caratteristica della pubblicità che, prevalentemente, è caratterizzata dall'ironia dalla battuta.

La deformazione dei valori costituisce la conseguenza della trasformazione della coscienza, che avviene sotto l'influsso dei cambiamenti globali nella cultura di quest'epoca. Il professor E. V. Socolov osserva: «lo sviluppo della cultura è collegato sia alla crescità dei valori che alla loro trasformazione qualitativa. I valori costituiscono l'essenza 
motivazionale della condotta dell'uomo e il loro sistema influisce direttamente sulla stabilità dei rapporti sociali, sull'intensità del lavoro, sulla direzione e le dimensioni dell'attività creativa. In questi sono sintetizzati, evidenziati e purificati i lati più sostanziali e significativi dell'esperienza umana e si sono depositati pensieri e sentimenti di molte generazioni. Perciò i valori appaiono come i fenomeni normativi che regolano la condotta, il sistema dei rapporti sociali e perfino la vita psichica» (Sokolov, E.V. (1972) Cultura e personalità: Nauka. pp. 90-91)

Secondo il ricercatore V.V.Bychkov il pensatore tedesco F.Nietzsche era uno dei primi filosofi del suo tempo che ha sentito la crisi della cultura e dell'arte e in gran parte le sue idee hanno influenzato la formazione dei fenomeni del periodo postmoderno. «F.Nietzsche ravvisava i motivi della crisi della cultura nella superiorità dell'intelligenza sull'istinto; nel culto dell'anima, della mente e dell'inizio spirituale; nel riconoscimento della prevalenza del mondo spirituale su quello materiale; nell'invenzione dell'idea di Dio. F.Nietzsche sosteneva "il rifiuto della dittatura e del culto della ragione e della morale tradizionale»; la soluzione «al di là del bene e del male», cioè il rifiuto delle valutazioni unidimensionali e identiche e dei valori stabili» (Bychkov V.V. (2004) Estetica: un libro di testo. Mosca: Gardariki. p. 313). II permissivismo, come credeva F.Nietzsche, è la particolarità distintiva dell'aristocrazia e della grandezza del uomo nuovo, chi si trova dall'altra parte dei valori universali.

«ll principio del rifiuto della ragione a favore dell'istinto, la priorità di assurdo, di paradossalità, relativismo di tutti i valori, permissivismo, processi caotici, fisiologismo ecc. sono diventati dominanti nella post-cultura» (Bychkov, V.V. (2004) Estetica: un libro di testo. Mosca: Gardariki. pp. 316-317). È necessario notare che con tutta la rigidità delle visioni del mondo di F.Nietzsche, molti ricercatori si basano attivamente sulle sue idee come fondamento teoretico provato con il tempo.

Uno dei primi che ha apprezzato il significato dell'approccio relativistico alla storia, era O.Spengler che nel suo libro «ll tramonto dell'Occidente» ha confrontato le scoperte di F.Nietzsche con quelle di N.Kopernik.

La post-cultura è caratterizzata, come già menzionato, dalla frammentazione dell'unità culturale, dall'attenzione alla personalità e al suo mondo interiore, dalla crescità delle opportunità della multivariazione del progresso, dall'incremento improvviso delle varietà culturali e quelle sociali. Ma lo sviluppo della post-cultura non è possibile senza mass media che la considerano il fattore e di conseguenza la post-cultura diventa cultura di massa.

Non è possibile parlare di cultura di massa senza rivolgersi alla pubblicità, a questo potentissimo strumento di fondazione della coscienza sociale.

Cultura di massa e pubblicità sono legati strettamente tra di loro. La pubblicità come elemento di cultura di massa forma la coscienza acritica e quella dei consumatori che è inerente alla società del consumerismo. Entrambe sono strumenti convenienti, tecnologici e psicologici per manipolare la coscienza pubblica. Secondo O.A.Feofanov «il legame tra la pubblicità e la cultura è immediato. Anzi la pubblicità stessa fa parte di «cultura di massa» ed i suoi principi si usano ampiamente in tutti i suoi generi. Un'altra particolarità comune di pubblicità e "cultura di massa» consiste nel fatto che entrambe hanno la capacità di trivializzare cioè semplificare e stabilire una media della coscienza di massa. Sono capaci di trasformare in banalità ogni valore e ogni contenuto e così ridurre a zero il suo senso sociale. Il sociologo americano U.Genri nel suo articolo «La pubblicita come il sistema filosofico» sottolinea che la pubblicità "porta via i valori e gradualmente li rende banali e alla fine contribuisce al riguardo indifferente e perfino cinico nei loro confronti». (Reid, H.G. (1974) Up the Mainstream: A Critigue of Ideologic in American Politics and Everyday Life. N. Y.: McKay. p. 123)

A questa conclusione arriva anche un ricercatore della pubblicità D.K.Busi: «Per imporre uno stile 0 un gusto definito la pubblicità ha dovuto distruggere gli oggetti originali. Sarà l'unica delle leggi principali di «cultura di massa...» (Buiv, G. (1968) Advertising: Its Cultural and Political Effects. Menneapolis. p. 60)

Questa vicinanza di pubblicità e "cultura di massa" porta molto spesso al punto in cui le stesse si chiedono aiuto di continuo. Per esaltare delle qualità speciali dei prodotti la pubblicità usa spesso gli strumenti e anche la tecnologia di «cultura di massa», ad esempio: delle canzoncine popolari; delle immagini tradizionali, diventate già mitiche, dei cartoni animati 0 dei fumetti. D'altra parte la «cultura di massa» usa molti meccanismi affidabili della pubblicità e le sue relazioni con i consumatori di massa; i suoi metodi tradizionali dell'influenza sulla coscienza collettiva, ad esempio: ripetitività, insolenza intenzionale, appello ai bisogni immaginari, relazione con le leggi di mercato, con quelle della domanda e dell'offerta, ma non con la mente.

«Con l'aiuto della pubblicita, la «cultura di massa» si diffonde non solo dentro uno o l'altro paese ma anche su scala globale» (Shestakov, V.P. Mitologia XX Secolo: La critica della teoria e della pratica del borghese "cultura di massa" accessibile [Online] /http://www.gumer.info/bibliotek_Buks/Culture/Shest/04.php/ (27 giugno, 2013)

In questo caso si osserva il parallelo tra gli strumenti e meccanismi che usa la pubblicità e le previsioni del filosofo tedesco chi rilevava nei suoi giudizi che riguardavano la crisi di cultura, la priorita dell'assurdo, della paradossalità, del relativismo di tutti i valori. 
II riflesso delle sue parole lo troviamo nella pubblicità moderna la sostanza di cui sono composte delle dichiarazioni di F.Nietzsche che non hanno ancora perso la loro attulità.

Avendo esaurito l'arsenale dei valori classici la pubblicità crea i nuovi valori immaginari che si chiamano consumistici. Come scrive il pubblicitario americano S. Iven, - «La creazione dei bisogni immaginari si è presentata come un fattore forte per la pubblicità moderna. La sparizione dei mercati di consumo tradizionali e delle abitudini rispettive, richiedeva che la gente facesse acquisti non per la soddisfazione dei suoi bisogni reali ma piuttosto per la soddisfazione dei bisogni della produzione industriale». (Reid, H.G. (1974) Up the Mainstream: A Critigue of Ideologic in American Politics and Everyday Life. N. Y.: McKay. p. 141)

Mediante la pubblicità, la «cultura di massa» è diventata un'industria enorme che si sforza di sostituire l'arte vera e la cultura vera. La «cultura di massa» crea la mitologia moderna e la pubblicità introduce questi miti dentro la coscienza di massa.

«La pubblicità come strumento e condizione necessaria di «cultura di massa» sostiene la sua esistenza e la sua diffusione. D'altra parte la «cultura di massa» rende la pubblicità raffinata e effettiva. L'alleanza tra pubblicita e «cultura di massa» è la legge del funzionamento della società consumistica». (Reid, H.G. (1974) Up the Mainstream: A Critigue of Ideologic in American Politics and Everyday Life. N. Y.: McKay. p. 141)

Dunque la pubblicità costruisce e sostiene l'esistenza della "cultura di massa" e anche costituisce la sua condizione necessaria. La «cultura di massa» per ottenere l'elevazione di efficienza, rende la pubblicità più raffinata e più ironica. (U.Eco)

Analizzando la specificità dell'influenza della pubblicità sulla società consumistica non si può non notare che la pubblicità usa molto spesso i simboli. Proprio il simbolo di qualsiasi epoca è una specie di catalizzatore. (J.M.Lotman) Vediamo le definizioni dei ricercatori riguardo al simbolo.

O.Spengler nel suo lavoro principale "II tramonto dell'Occidente" formula il concetto di carattere filosofico-culturale basato sulle nozioni di cultura e di civiltà.

II concetto storico-culturale di O.Spengler si costruisce sui confronti, sulla correlazione e perlopiù sulla contrapposizione paradossale di «cultura» e «civiltà». Nello stesso tempo la critica giusta delle teorie tradizionali, la ricerca della situazione socio-culturale moderna, la prova di analizzare gli origini della crisi culturale rendono il libro «ll tramonto dell'Occidente» molto attuale.

Dal punto di vista dello scienziato, la chiave della comprensione della storia mondiale della cultura è il simbolo. Lui scrive che per ricreare lo spirito di un'epoca non è sufficiente solo illustrare la storia attraverso altri avvenimenti, monumenti, opere d'arte.

È necessario chiarire la correlazione più profonda tra le forme culturali che si trovano nel simbolo. «Popoli, lingue e epoche, battaglie e idee, paesi e Dei, arti e opere, scienze, rapporti giuridici, forme economiche, concezioni del mondo, gente famosa e eventi grandi; tutti questi sono simboli e sono soggetti ad interpretazione» (Spengler, O. (1993) II tramonto dell'Occidente. Saggi sulla morfologia della storia mondiale. Mosca: Nauka)

Ogni cultura e ogni epoca hanno il proprio simbolo, il proprio segno rappresentativo. Proprio simboli e segni formano l'immagine della cultura e in un certo modo si mettono insieme in un tutto indivisibile che ricrea l'aspetto di un'epoca.

S.Freud, E.Fromm, J. Kristeva e gli altri esaminano il simbolo in qualità di unica possibilità mediata della rivelazione degli inizi incoscienti nella psiche umana e nella cultura.

Un considerevole interesse suscitano le idee di K.G. Jung sull'esistenza delle immagini-simboli universali dell'incoscio collettivo (come gli archetipi).

Nei limiti dell'interpretazione del simbolo nella cultura è rapppresentata l'idea principale di A.F. Losev che ha fatto una delle ricerche più profonde sul simbolo, basandosi sulla tradizione platonica nella cultura europea. Per di più ha sintetizzato e rielaborato le opinioni, a quei tempi moderne, di P.Natorp, G.Kogen, E.Gusserl, avendo riflesso quegli indirizzi del pensiero filosofico che hanno formulato nei limiti della visione simbolica del mondo di P.Florenskije E.Cassirer. A.F. Losev considera simbolo il fondamento ontologico della cultura, il principio organizzativo della vita umana, che permette di creare l'universo. La corrente simbolica della filosofia di cultura ha permesso di trovare i nuovi metodi dello studio della cultura tramite i suoi simboli e ha determinato la tendenza di sviluppo della comprensione del problema nei limiti del moderno discorso culturologico.

E così il potere dell'influenza della pubblicità con l'uso dei simboli cresce notevolmente. La causa di ciò sta nel potenziale grande del simbolo, la valutazione di cui è stata riportata prima. Inoltre il simbolo pubblicitario è una parte significativa dell'estensione comunicativa nella cultura postmoderna, il modo per rappresentare la sua importanza e il suo stereotipo. 
Come è già stato menzionato, il XX secolo si è distinto per cambiamenti seri nella cultura artistica. La tappa della svolta è avvenuta nei anni 50, quando hanno cominciato a cancellarsi i limiti tra pubblicità e cultura. I mezzi artistici esistenti non riuscivano a trasmettere gli umori della società, che erano legati alla fioritura della cultura del consumo.

La soluzione è stata inaspettata: trascurando l'inerpretazione, creare la realtà della società moderna, senza valutazione, analisi, ironia 0 emozione. L'attenzione dei pittori si rivolge alla pubblicità come indicatore singolare sociale, che è di per sè piena delle necessarie emozioni false, delle immagini, della valutazione. L'influenza della pubblicità è stata rafforzata dall'uso del simbolo, che era un potente mezzo espressivo.

«...Quando si usa l'epiteto «pop» nell'arte, si tende all'associazione con diversi aspetti esteriori della società. La pop-art compie un lavoro equilibrato tra i più euforici, progressivi tipi di un'epoca da una parte e la prospettiva pessimistica catastrofica dall'altra parte. L'aumento della comercializzazione che trapassa la nostra realtà sociale, ha fatto ridurre nell'ambiente informativo l'idea dei valori tipo «bene, verità e bellezza». Le norme di cultura stampano le immagini nelle persone e negli oggetti, nella natura e nella tecnologia...» (Osterwold , T. (2011) Pop Art. Köln: Taschen, P. 6) - ritiene T. Ostervold.

II simbolo dell'arte «alta» si assume per fondamento di tali opere e tramite la pubblicità varca il confine del sacrale e diventa pure di massa. francobolli.

Così gli unici capolavori singolari per esempio «Mona Lisa» sono diventati delle riproduzioni non più preziosi dei

Avendo segnato l'oggetto dell'arte come il prodotto del mercato il notevole rappresentante della Pop-Art A. Warhol continua a coltivare la sua partecipazione di massa, ottenendo l'effetto di «depersonalizzazione» di questo prodotto attraverso la copiatura dell'immagine nei limiti di un lavoro singolo o di una serie di questi. «Tutti i suoi lavori successivi ed i più famosi - sono le immagini del segno di dollaro, della minestra in scatola, delle bottiglie di Coca-Cola copiate molte volte, le foto di celebrità riprodotti gran numero di volte».

Tra l'altro tale deprezzamento entra in contraddizione con la missione dell'arte stessa. A. Warhol è riuscito non solo a far somigliare le sue opere a oggetti di «cultira di massa» ma anche ad innalzare questi oggetti a livello dell'arte.

La minestra in scatola diventa un art-simbolo immortale. «Quando si sforzano di dissacrare la pratica, la societa invece la consacra di più, - crede il culturologo francese $\mathrm{J}$. Baudrillard, - E tutto finisce così che la loro prova, la più radicale di tutte quelle precedenti, della secolarizzazione dell'arte nei suoi temi e nella sua pratica apre la strada dell'esaltazione e della certezza del sacro nell'arte mai vista prima...» (Baudrillard, J. (2006) La società dei consumi. I suoi miti e le strutture. M.: La Rivoluzione culturale; Repubblica, p. 155)

Sembrava che la pubblicità avesse fatto l'impossibile: ha dato il prestigio, il valore a molto di quello che non era una vera realizzazione artistica, anzi non si è pensato neanche come un'opera d'arte, ma in questa qualità si vendeva e si comprava bene. È sucessa una cosa interessante: «...la protesta antiborghese è stata utilizzata dalla stessa realtà borghese...» (Ikonnikova, S.N., Bolshakov, V.P. (2010) La teoria della cultura: il manuale. San Pietroburgo: Peter. p. 484)

Siamo giunti alla conclusione che cultura e pubblicità si trovano tra di loro in una stretta interconnesione, e in più hanno l'effetto dell'influenza reciproca, dell'interpenetrazione.

Tramite la pubblicità, la «cultura di massa» è diventata un'industria enorme che ha respinto la vera arte e la vera cultura. La pubblicità come strumento dà alla «cultura di massa» la possibilità di esistere e di volgarizzarsi. A sua volta la «cultura di massa» rende la pubblicità più efficace. E la pubblicità usando una moltitudine di simboli acquisisce maggior influenza sulla società moderna. Pubblicità e "cultura di massa" formano la legge di funzionamento della società moderna, della «società di consumo».

\section{References}

Baudrillard J. (2006) La società dei consumi. I suoi miti e le strutture. M.: La Rivoluzione culturale; Repubblica , p. 155

Buiv, G. (1968) Advertising: Its Cultural and Political Effects. Menneapolis. p. 60

Bychkov V.V. (2004) Estetica: un libro di testo. Mosca: Gardariki. pp. 313-317

Ikonnikova, S.N., Bolshakov, V.P. (2010) La teoria della cultura: il manuale. San Pietroburgo: Peter. p. 484

Kagan, M.S. (1997) Estetica come scienza filosofica. San Pietroburgo: TOO TK "Petropolis". p. 523

Osterwold , T. (2011) Pop Art. Köln: Taschen, p. 6

Reid, H.G. (1974) Up the Mainstream: A Critigue of Ideologic in American Politics and Everyday Life. N. Y.: McKay. pp. 123-141

Sokolov, E.V. (1972) Cultura e personalità: Nauka. pp. 90-91

Spengler, O. (1993) II tramonto dell'Occidente. Saggi sulla morfologia della storia mondiale. Mosca: Nauka

Shestakov, V.P. Mitologia XX Secolo: La critica della teoria e della pratica del borghese "cultura di massa" accessibile [Online] /http://www.gumer.info/bibliotek_Buks/Culture/Shest/04.php/ (27 giugno, 2013) 